\title{
Distant total irregularity strength of graphs via random vertex ordering
}

\author{
Jakub Przybyło ${ }^{1,2}$ \\ AGH University of Science and Technology, al. A. Mickiewicza 30, 30-059 Krakow, Poland
}

\begin{abstract}
Let $c: V \cup E \rightarrow\{1,2, \ldots, k\}$ be a (not necessarily proper) total colouring of a graph $G=(V, E)$ with maximum degree $\Delta$. Two vertices $u, v \in V$ are sum distinguished if they differ with respect to sums of their incident colours, i.e. $c(u)+\sum_{e \ni u} c(e) \neq$ $c(v)+\sum_{e \ni v} c(e)$. The least integer $k$ admitting such colouring $c$ under which every $u, v \in V$ at distance $1 \leq d(u, v) \leq r$ in $G$ are sum distinguished is denoted by $\operatorname{ts}_{r}(G)$. Such graph invariants link the concept of the total vertex irregularity strength of graphs with so called 1-2-Conjecture, whose concern is the case of $r=1$. Within this paper we combine probabilistic approach with purely combinatorial one in order to prove that $\operatorname{ts}_{r}(G) \leq(2+o(1)) \Delta^{r-1}$ for every integer $r \geq 2$ and each graph $G$, thus improving the previously best result: $\operatorname{ts}_{r}(G) \leq 3 \Delta^{r-1}$.
\end{abstract}

Keywords: total vertex irregularity strength of a graph, 1-2 Conjecture, $r$-distant total irregularity strength of a graph

\section{Introduction}

The cornerstone of the field of vertex distinguishing graph colourings is the graph invariant called irregularity strength. For a graph $G=(V, E)$ it is usually denoted by $s(G)$ and can be defined as the least integer $k$ so that we may construct an irregular multigraph, i.e. a multigraph with pairwise distinct degrees of all vertices, of $G$ by multiplying its edges, each at most $k$ times (including the original one), see [8]. This study thus originated from the basic fact that no graph $G$ with more than one vertex is irregular itself and related research on possible alternative definitions of an irregular graph, see e.g. 7]. Equivalently, $s(G)$ is also defined as the least $k$ so that there exists an edge colouring $c: E \rightarrow\{1,2, \ldots, k\}$ such that for every pair $u, v \in E, u \neq v$, the sum of colours incident with $u$ is distinct from the sum of colours incident with $v$. Note that $s(G)$ exists only for graphs without isolated edges and with at most one isolated

\footnotetext{
Email address: jakubprz@agh.edu.pl, phone: 048-12-617-46-38, fax: 048-12-617-31-65 (Jakub Przybyło)

${ }^{1}$ Financed within the program of the Polish Minister of Science and Higher Education named "Iuventus Plus" in years 2015-2017, project no. IP2014 038873.

${ }^{2}$ Partly supported by the Polish Ministry of Science and Higher Education. 
vertex. It is known that $s(G) \leq n-1$, where $n=|V|$, for all such graphs, except for $K_{3}$, see [1, 21]. This tight upper bound can however be improved in the case of graphs with minimum degree $\delta \geq 1$ to $s(G) \leq 6\left\lceil\frac{n}{\delta}\right\rceil$ (what yields a better result whenever $\delta>12$ and for $\delta \in[7,12]$ if $n$ is larger than a small constant dependent on $\delta$ ), see [16], and to $s(G) \leq(4+o(1)) \frac{n}{\delta}+4$ for graphs with $\delta \geq n^{0.5} \ln n$, see [19]. Many interesting results, concepts and open problems concerning this graph invariant can also be found e.g. in 6, 9, 10, 11, 12, 13, 16, 18, 25], and many others.

In [5], Bača et al. introduced a total version of the concept above. Given any graph $G=(V, E)$ and a (not necessarily proper) total colouring $c: V \cup E \rightarrow\{1,2, \ldots, k\}$, let

$$
w_{c}(v):=c(v)+\sum_{u \in N(v)} c(u v)
$$

denote the weight of any vertex $v \in V$, which shall also be called the sum at $v$ and denoted simply by $w(v)$ in cases when $c$ is unambiguous from context. The least $k$ for which there exists such colouring with $w(u) \neq w(v)$ for every $u, v \in V, u \neq v$, is called the total vertex irregularity strength of $G$ and denoted by $\operatorname{tvs}(G)$. In [5], among others, it was proved that for every graph $G$ with $n$ vertices, $\left\lceil\frac{n+\delta}{\Delta+1}\right\rceil \leq \operatorname{tvs}(G) \leq n+\Delta-2 \delta+1$. Up to know the best upper bounds (for graphs with $\delta>3$ ) assert that $\operatorname{tvs}(G) \leq 3\left\lceil\frac{n}{\delta}\right\rceil+1$, see [3], and $\operatorname{tvs}(G) \leq(2+o(1)) \frac{n}{\delta}+4$ for $\delta \geq n^{0.5} \ln n$, see [20]. Many other results e.g. for particular graph families can also be found in [4, 22, 26, 29] and other papers.

In this article we consider a distant generalization of $\operatorname{tvs}(G)$ from 23], motivated among others by the study on distant chromatic numbers, see e.g. 17] for a survey concerning these. For any positive integer $r$, two distinct vertices at distance at most $r$ in $G$ shall be called $r$-neighbours. We denote by $N^{r}(v)$ the set of all $r$-neigbours of any $v \in V$ in $G$, and set $d^{r}(v)=\left|N^{r}(v)\right|$. The least integer $k$ for which there exists a total colouring $c: V \cup E \rightarrow\{1,2, \ldots, k\}$ such that there are no $r$-neighbours $u, v$ in $G$ which are in conflict, i.e. with $w(u)=w(v)$ (cf. (11)), we call the $r$-distant total irregularity strength of $G$, and denote by $\operatorname{ts}_{r}(G)$. It is known that $\operatorname{ts}_{r}(G) \leq 3 \Delta^{r-1}$ for every graph $G$, see [23], also for a comment implying that a general upper bound for $\operatorname{ts}_{r}(G)$ cannot be (much) smaller than $\Delta^{r-1}$. In this paper we combine the probabilistic method with algorithmic approach similar to those in e.g. 3, 15, 20, 23] to prove that in fact $\operatorname{ts}_{r}(G) \leq(2+o(1)) \Delta^{r-1}$ (for $\left.r \geq 2\right)$.

Theorem 1. For every integer $r \geq 2$ there exists a constant $\Delta_{0}$ such that for each graph $G$ with maximum degree $\Delta \geq \Delta_{0}$,

$$
\operatorname{ts}_{r}(G) \leq 2 \Delta^{r-1}+3 \Delta^{r-\frac{4}{3}} \ln ^{2} \Delta+4,
$$

hence

$$
\operatorname{ts}_{r}(G) \leq(2+o(1)) \Delta^{r-1}
$$

for all graphs.

It is also worth mentioning that the case of $r=1$ was introduced and considered separately in [27], where the well known 1-2-Conjecture concerning this invariant was introduced. It is known that $\mathrm{ts}_{1}(G) \leq 3$ for all graphs, see Theorem 2.8 in [15], even in case of a natural list generalization of the problem, see [30], though it is believed that the upper bound of 2 should make the optimal general upper bound in both cases, see [27, 28, 31]. 
We also refer a reader to [24] to see an improvement of a similar probabilistic flavor for the upper bound from [23] on the correspondent of $\operatorname{ts}_{r}(G)$ concerning the case of edge colourings exclusively.

\section{Probabilistic Tools}

We shall use probabilistic approach in the first part of the proof of Theorem 1, basing on the Lovász Local Lemma, see e.g. [2], combined with the Chernoff Bound, see e.g. 14] (Th. 2.1, page 26). We recall these below.

Theorem 2 (The Local Lemma). Let $A_{1}, A_{2}, \ldots, A_{n}$ be events in an arbitrary probability space. Suppose that each event $A_{i}$ is mutually independent of a set of all the other events $A_{j}$ but at most $D$, and that $\operatorname{Pr}\left(A_{i}\right) \leq p$ for all $1 \leq i \leq n$. If

$$
e p(D+1) \leq 1
$$

then $\operatorname{Pr}\left(\bigcap_{i=1}^{n} \overline{A_{i}}\right)>0$.

Theorem 3 (Chernoff Bound). For any $0 \leq t \leq n p$,

$$
\operatorname{Pr}(\operatorname{BIN}(n, p)>n p+t)<e^{-\frac{t^{2}}{3 n p}} \text { and } \operatorname{Pr}(\operatorname{BIN}(n, p)<n p-t)<e^{-\frac{t^{2}}{2 n p}} \leq e^{-\frac{t^{2}}{3 n p}}
$$

where $\operatorname{BIN}(n, p)$ is the sum of $n$ independent Bernoulli variables, each equal to 1 with probability $p$ and 0 otherwise.

Note that if $X$ is a random variable with binomial distribution $\operatorname{BIN}(n, p)$ where $n \leq k$, then we may still apply the Chernoff Bound above, even if we do not know the exact value of $n$, to prove that $\operatorname{Pr}(X>k p+t)<e^{-\frac{t^{2}}{3 k p}}($ for $t \leq\lfloor k\rfloor p)$.

\section{Proof of Theorem 1}

Fix an integer $r \geq 2$. Within our proof we shall not specify $\Delta_{0}$. Instead, we shall assume that $G=(V, E)$ is a graph with sufficiently large maximum degree $\Delta$, i.e. large enough so that all inequalities below are fulfilled.

We first partition $V$ into a subset of vertices with relatively small degrees and a subset of those with big degrees:

$$
\begin{aligned}
& S=\left\{u \in V: d(u) \leq \Delta^{\frac{2}{3}}\right\} \\
& B=\left\{u \in V: d(u)>\Delta^{\frac{2}{3}}\right\}
\end{aligned}
$$

Moreover, for every $v \in V$, we denote: $S(v)=N(v) \cap S, s(v)=|S(v)|, B(v)=N(v) \cap B$, $b(v)=|B(v)|$.

Now we randomly order the vertices of $V$ into a sequence. For this goal, associate with every vertex $v \in V$ a random variable $X_{v} \sim U[0,1]$ having the uniform distribution on $[0,1]$ where all these random variables $X_{v}, v \in V$ are independent, or in other words pick a (real) number uniformly at random from the interval $[0,1]$ and associate it with $v$ for every $v \in V$. Note that with probability one all these numbers are pairwise distinct. 
In such a case, these independent random variables uniquely define a natural ordering $v_{1}, v_{2}, \ldots, v_{n}$ of the vertices in $V$ where $X_{v_{i}}<X_{v_{j}}$ if and only if $1 \leq i<j \leq n$.

For every vertex $v \in V$, any its neighbour or $r$-neighbour $u$ which precedes $v$ in the obtained ordering of the elements of $V$ shall be called a backward neighbour or $r$ neighbour, resp., of $v$. Analogously, the remaining ones shall be called forward neighbours or r-neighbours, resp., of $v$, while the edges joining $v$ with its forward or backward neighbours shall be referred to as forward or backward edges, resp., as well. Also, for any subset $W \subset V$, let $N_{-}(v), N_{-}^{r}(v), N_{W}^{r}(v)$ denote the sets of all backward neighbours, backward $r$-neighbours and $r$-neighbours in $W$ of $v$, respectively. Set $d_{-}^{r}(v)=\left|N_{-}^{r}(v)\right|$, $d_{W}^{r}(v)=\left|N_{W}^{r}(v)\right|$, and let $b_{-}(v)$ denote the number of backward neighbours of $v$ which belong to $B(v)$.

Denote $D(v)=\sum_{u \in N(v)} d(u)$ and note that $d^{r}(v) \leq D(v) \Delta^{r-2} \leq d(v) \Delta^{r-1}$.

Let us also partition $V$ into a subset $I$ consisting of initial vertices of the obtained sequence and the remaining part $R$ :

$$
\begin{aligned}
& I=\left\{v: X_{v}<\frac{\ln \Delta}{\Delta^{\frac{1}{3}}}\right\} \\
& R=\left\{v: X_{v} \geq \frac{\ln \Delta}{\Delta^{\frac{1}{3}}}\right\} .
\end{aligned}
$$

Lemma 4. With positive probability, the obtained ordering has the following features for every vertex $v$ in $G$ with $b(v) \geq \Delta^{\frac{1}{3}} \ln \Delta$ :

$$
\begin{aligned}
& F_{1}: d_{I}^{r}(v) \leq 2 d(v) \Delta^{r-\frac{4}{3}} \ln \Delta ; \\
& F_{2}: \text { if } v \in R, \text { then: } b_{-}(v) \geq X_{v} b(v)-\sqrt{X_{v} b(v)} \ln \Delta ; \\
& F_{3}: \text { if } v \in R, \text { then: } d_{-}^{r}(v) \leq X_{v} D(v) \Delta^{r-2}+\sqrt{X_{v} D(v) \Delta^{r-2}} \ln \Delta .
\end{aligned}
$$

Proof. For every vertex $v \in V$ of degree $d$ in $G$ and with $b(v) \geq \Delta^{\frac{1}{3}} \ln \Delta$ (hence also $\left.d \geq \Delta^{\frac{1}{3}} \ln \Delta\right)$, let $A_{v, 1}$ denote the event that $d_{I}^{r}(v)>2 d \Delta^{r-\frac{4}{3}} \ln \Delta$, let $A_{v, 2}$ be the event that $v$ belongs to $R$ and $b_{-}(v)<X_{v} b(v)-\sqrt{X_{v} b(v)} \ln \Delta$, and let $A_{v, 3}$ denote the event that $v$ belongs to $R$ and $d_{-}^{r}(v)>X_{v} D(v) \Delta^{r-2}+\sqrt{X_{v} D(v) \Delta^{r-2}} \ln \Delta$.

As $\left|N^{r}(v)\right| \leq d \Delta^{r-1}$ and for each $u \in N^{r}(v)$, the probability that $u$ belongs to $I$ equals $\frac{\ln \Delta}{\Delta^{\frac{1}{3}}}$, then by the Chernoff Bound (and the comment below it),

$$
\begin{aligned}
\operatorname{Pr}\left(A_{v, 1}\right) & \leq \operatorname{Pr}\left(d_{I}^{r}(v)>d \Delta^{r-\frac{4}{3}} \ln \Delta+\sqrt{d \Delta^{r-\frac{4}{3}} \ln \Delta} \ln \Delta\right) \\
& <e^{-\frac{d \Delta^{r-\frac{4}{3}} \ln ^{3} \Delta}{3 d \Delta^{r-\frac{4}{3}} \ln \Delta}}=\Delta^{-\frac{\ln \Delta}{3}}<\frac{1}{\Delta^{3 r}}
\end{aligned}
$$

Subsequently note that for any $x \in[0,1]$ :

$$
\begin{aligned}
& \operatorname{Pr}\left(b_{-}(v)<X_{v} b(v)-\sqrt{X_{v} b(v)} \ln \Delta \mid X_{v}=x\right) \\
= & \operatorname{Pr}(\operatorname{BIN}(b(v), x)<x b(v)-\sqrt{x b(v)} \ln \Delta) \\
< & \frac{1}{\Delta^{3 r}},
\end{aligned}
$$


where the last inequality follows by the Chernoff Bound if $\sqrt{x b(v)} \ln \Delta \leq x b(v)$, while it is trivial otherwise. Hence,

$$
\operatorname{Pr}\left(A_{v, 2}\right) \leq \operatorname{Pr}\left(b_{-}(v)<X_{v} b(v)-\sqrt{X_{v} b(v)} \ln \Delta\right) \leq \int_{0}^{1} \frac{1}{\Delta^{3 r}} d x=\frac{1}{\Delta^{3 r}} .
$$

For the sake of analyzing $A_{v, 3}$, note now first that for $x \in\left[0, \frac{\ln \Delta}{\Delta^{\frac{1}{3}}}\right)$,

$$
\operatorname{Pr}\left(d_{-}^{r}(v)>X_{v} D(v) \Delta^{r-2}+\sqrt{X_{v} D(v) \Delta^{r-2}} \ln \Delta \wedge v \in R \mid X_{v}=x\right)=0 .
$$

On the other hand, analogously as above, for $x \in\left[\frac{\ln \Delta}{\Delta^{\frac{1}{3}}}, 1\right]$ :

$$
\begin{aligned}
& \operatorname{Pr}\left(d_{-}^{r}(v)>X_{v} D(v) \Delta^{r-2}+\sqrt{X_{v} D(v) \Delta^{r-2}} \ln \Delta \wedge v \in R \mid X_{v}=x\right) \\
\leq & \operatorname{Pr}\left(\operatorname{BIN}\left(D(v) \Delta^{r-2}, x\right)>x D(v) \Delta^{r-2}+\sqrt{x D(v) \Delta^{r-2}} \ln \Delta\right) \\
< & \frac{1}{\Delta^{3 r}},
\end{aligned}
$$

where the last inequality follows by the Chernoff Bound, as $x \geq \frac{\ln \Delta}{\Delta^{\frac{1}{3}}}$ and $b(v) \geq \Delta^{\frac{1}{3}} \ln \Delta$ (where $D(v) \geq b(v) \Delta^{\frac{2}{3}}$ ) imply that $\sqrt{x D(v) \Delta^{r-2}} \ln \Delta \leq x D(v) \Delta^{r-2}$. Hence, by (5) and (6),

$$
\operatorname{Pr}\left(A_{v, 3}\right) \leq \int_{0}^{1} \frac{1}{\Delta^{3 r}} d x=\frac{1}{\Delta^{3 r}} .
$$

Note that each event $A_{v, i}$ is mutually independent of all other events except those $A_{u, j}$ with $u$ at distance at most $2 r$ from $v, i, j \in\{1,2,3\}$, i.e., at most $3 \Delta^{2 r}+2$ events. Thus, as by (3), (4) and (7), the probability of each such event is bounded from above by $\Delta^{-3 r}$, by the Lovász Local Lemma, with positive probability none of the events $A_{v, i}$ with $v \in V$ (and $\left.b(v) \geq \Delta^{\frac{1}{3}} \ln \Delta\right)$ and $i \in\{1,2,3\}$ appears.

Let $v_{1}, v_{2}, \ldots, v_{n}$ be the ordering of the vertices of $V$ guaranteed by Lemma 4 . Set

$$
K=\Delta^{r-1}+\left\lceil\Delta^{r-\frac{4}{3}} \ln ^{2} \Delta\right\rceil \text { and } k=\left\lceil\Delta^{r-\frac{4}{3}} \ln ^{2} \Delta\right\rceil,
$$

and assign initial colour 1 to all the vertices and initial colour $K+1$ to all the edges of $G$. We shall construct our final colouring $f: V \cup E \rightarrow\{1,2, \ldots, 2 K+k+1\}$ using an algorithm within which we shall be analyzing the consecutive vertices in the ordering (starting from $v_{1}$ ). Denote by $c_{t}(a)$ the contemporary colour of every $a \in V \cup E$ at every stage of the ongoing algorithm (hence initially $c_{t}(v)=1$ and $c_{t}(e)=K+1$ for every $v \in V$ and $e \in E)$. The final target sum of every vertex $v \in V, w_{f}(v)$, shall be chosen the moment $v$ is analyzed. For every $v \in V$, ever since $w_{f}(v)$ is chosen, we shall require so that

$$
0 \leq w_{f}(v)-w_{c_{t}}(v) \leq K
$$

We shall admit at most two alterations of the colour for every edge in $E$ - only when any of its ends is being analyzed (vertex colours shall be adjusted at the end of the algorithm). For every currently analyzed vertex $v$ and its neighbour $u \in N(v)$, we admit the following alterations of the colour of $e=u v$ (the moment $v$ is analyzed): 
- adding $0,1, \ldots, K-1$ or $K$ if $e$ is a forward edge of $v, v \in S$ and $u \in B$,

- adding $0,1, \ldots, k-1$ or $k$ if $e$ is a forward edge of $v$ (and $v \in B$ or $u \in S$ ),

- adding $-K,-K+1, \ldots, K-1$ or $K$ if $e$ is a backward edge of $v$ and $u \in B$,

- adding $-k,-k+1, \ldots, k-1$ or $k$ if $e$ is a backward edge of $v$ and $u \in S$,

so that afterwards (8) is fulfilled for every vertex $u \in N_{-}(v)$ and for $v$ (after processing all edges incident with $v$ ). Note that the admitted alterations guarantee that $c_{t}(e) \in$ $\{1,2, \ldots, 2 K+k+1\}$ for every $e \in E$ at every stage of the construction.

Suppose we are about to analyze a vertex $v=v_{i}, i \in\{1,2, \ldots, n\}$, and thus far all our requirements have been fulfilled. We shall show that in every case the admitted alterations on the edges incident with $v$ provide us more options for $w_{c_{t}}(v)$ than there are backward $r$-neighbours of $v$, and hence one of this options can be fixed as $w_{f}(v)$ so that this value is distinct from every $w_{f}(u)$ already fixed for any $u \in N_{-}^{r}(v)$. Denote the degree of $v$ by $d$, and assume that $d>0$ (otherwise, we set $w_{f}(v)=1$ ):

- If $v \in I, v \in B$ and $b(v) \geq \Delta^{\frac{1}{3}} \ln \Delta$, then the admitted alterations provide at least $d k \geq d \Delta^{r-\frac{4}{3}} \ln ^{2} \Delta$ available options for $w_{c_{t}}(v)$. As by $F_{1}$ (from Lemma 4), $\left|N_{-}^{r}(v)\right| \leq d_{I}^{r}(v) \leq 2 d \Delta^{r-\frac{4}{3}} \ln \Delta<d \Delta^{r-\frac{4}{3}} \ln ^{2} \Delta$, at least one of these available options is distinct from all $w_{f}(u)$ with $u \in N_{-}^{r}(v)$.

- If $v \in S$, then the admitted alterations provide at least $s(v) k+b(v) K \geq s(v) \Delta^{r-\frac{4}{3}} \ln ^{2} \Delta+$ $b(v)\left(\Delta^{r-1}+\Delta^{r-\frac{4}{3}} \ln ^{2} \Delta\right)$ available options for $w_{c_{t}}(v)$. On the other hand, $\left|N_{-}^{r}(v)\right| \leq$ $d^{r}(v) \leq D(v) \Delta^{r-2} \leq\left(s(v) \Delta^{\frac{2}{3}}+b(v) \Delta\right) \Delta^{r-2}$, hence at least one of these available options is distinct from all $w_{f}(u)$ with $u \in N_{-}^{r}(v)$.

- If $v \in B$ and $b(v)<\Delta^{\frac{1}{3}} \ln \Delta$, then the admitted alterations provide at least $d k \geq$ $d \Delta^{r-\frac{4}{3}} \ln ^{2} \Delta$ available options for $w_{c_{t}}(v)$. On the other hand, analogously as in the case above, $\left|N_{-}^{r}(v)\right| \leq d^{r}(v) \leq s(v) \Delta^{\frac{2}{3}} \Delta^{r-2}+b(v) \Delta^{r-1}<d \Delta^{r-\frac{4}{3}}+\Delta^{r-\frac{2}{3}} \ln \Delta<$ $d \Delta^{r-\frac{4}{3}} \ln ^{2} \Delta$, as $v \in B$ implies that $d \geq \Delta^{\frac{2}{3}}$. We thus have at least one option available for $v$ distinct from all $w_{f}(u)$ with $u \in N_{-}^{r}(v)$.

- If $v \in R, v \in B$ and $b(v) \geq \Delta^{\frac{1}{3}} \ln \Delta$, then by $F_{2}$ the number of available options for $w_{c_{t}}(v)$ via admitted alterations of colours of the edges incident with $v$ is not smaller than:

$$
\begin{aligned}
b_{-}(v) K+\left(d-b_{-}(v)\right) k & \geq b_{-}(v) \Delta^{r-1}+d \Delta^{r-\frac{4}{3}} \ln ^{2} \Delta \\
& \geq\left(X_{v} b(v)-\sqrt{X_{v} b(v)} \ln \Delta\right) \Delta^{r-1}+d \Delta^{r-\frac{4}{3}} \ln ^{2} \Delta \\
& \geq X_{v} b(v) \Delta^{r-1}-\sqrt{d} \Delta^{r-1} \ln \Delta+d \Delta^{r-\frac{4}{3}} \ln ^{2} \Delta \\
& \geq X_{v} b(v) \Delta^{r-1}+d \Delta^{r-\frac{4}{3}} \ln ^{2} \Delta-d \Delta^{r-\frac{4}{3}} \ln \Delta
\end{aligned}
$$

(where the last inequality follows by the fact that $d \geq \Delta^{\frac{2}{3}}$ ). This number is however greater than the number of backward $r$-neighbours of $v$, as by $F_{3}$,

$$
\begin{aligned}
&\left|N_{-}^{r}(v)\right| \leq X_{v} D(v) \Delta^{r-2}+\sqrt{X_{v} D(v) \Delta^{r-2}} \ln \Delta \\
& \leq X_{v}\left(b(v) \Delta+s(v) \Delta^{\frac{2}{3}}\right) \Delta^{r-2}+\sqrt{d \Delta^{r-1}} \ln \Delta \\
& \leq X_{v} b(v) \Delta^{r-1}+d \Delta^{r-\frac{4}{3}}+d \Delta^{r-\frac{4}{3}} \ln \Delta . \\
& 6
\end{aligned}
$$


Thus in all cases there is at least one available sum, say $w^{*}$, for $v$ which is distinct from all $w_{f}(u)$ with $u \in N_{-}^{r}(v)$. We then set $w_{f}(v)=w^{*}$ and perform the admitted alterations on the edges incident with $v$ so that $w_{c_{t}}(v)=w^{*}$ afterwards.

By our construction, after analyzing $v_{n}$, all $w_{f}\left(v_{i}\right)$ are fixed for $i=1, \ldots, n$ so that $w_{f}(u) \neq w_{f}(v)$ whenever $u$ and $v$ are $r$-neighbours in $G$ and (8) holds for every $v \in V$. We then modify (if necessary) the colour of every vertex $v$ by adding to it the integer $w_{f}(v)-w_{c_{t}}(v)$, completing the construction of the desired total colouring $f$ of $G$ (by setting $f(a)=c_{t}(a)$ for every $a \in V \cup E$ afterwards). Note that $1 \leq f(e) \leq 2 K+k+1$ for every $e \in E$ and, by (8), $1 \leq f(v) \leq K+1$ for every $v \in V$, hence the thesis follows.

\section{Remarks}

We have put an effort to optimize the second order term from the upper bound in (2), up to a constant and a power in the logarithmic factor, which could still be slightly improved (at the cost of the clarity of presentation). Nevertheless, some multiplicative poly-logarithmic (in $\Delta$ ) factor seems unavoidable in this term within our approach.

We conclude by posing a conjecture, which to our believes expresses a true asymptotically optimal upper bound for the investigated parameters.

Conjecture 5. For every integer $r \geq 2$ and each graph $G$ with maximum degree $\Delta$,

$$
\mathrm{ts}_{r}(G) \leq(1+o(1)) \Delta^{r-1} .
$$

\section{References}

[1] M. Aigner, E. Triesch, Irregular assignments of trees and forests, SIAM J. Discrete Math. 3(4) (1990) 439-449.

[2] N. Alon, J.H. Spencer, The Probabilistic Method, 2nd edition, Wiley, New York, 2000.

[3] M. Anholcer, M. Kalkowski, J. Przybyło, A new upper bound for the total vertex irregularity strength of graphs, Discrete Math. 309 (2009) 6316-6317.

[4] M. Anholcer, M. Karoński, F. Pfender, Total Vertex Irregularity Strength of Forests, arXiv:1103.2087

[5] M. Bača, S. Jendrol̆, M. Miller, J. Ryan, On Irregular Total Labelings, Discrete Math. 307 (2007) $1378-1388$.

[6] T. Bohman, D. Kravitz, On the irregularity strength of trees, J. Graph Theory 45 (2004) 241-254.

[7] G. Chartrand, P. Erdős, O.R. Oellermann, How to Define an Irregular Graph, College Math. J. 19(1) (1988) 36-42.

[8] G. Chartrand, M.S. Jacobson, J. Lehel, O.R. Oellermann, S. Ruiz, F. Saba, Irregular networks, Congr. Numer. 64 (1988) 197-210.

[9] B. Cuckler, F. Lazebnik, Irregularity Strength of Dense Graphs, J. Graph Theory 58(4) (2008) 299-313.

[10] J.H. Dinitz, D.K. Garnick, A. Gyárfás, On the irregularity strength of the $m \times n$ grid, J. Graph Theory 16 (1992) 355-374.

[11] R.J. Faudree, M.S. Jacobson, J. Lehel, R. Schelp, Irregular networks, regular graphs and integer matrices with distinct row and column sums, Discrete Math. 76 (1989) 223-240.

[12] R.J. Faudree, J. Lehel, Bound on the irregularity strength of regular graphs, Colloq Math Soc Jańos Bolyai, 52, Combinatorics, Eger North Holland, Amsterdam, (1987), 247-256.

[13] A. Frieze, R.J. Gould, M. Karoński, F. Pfender, On Graph Irregularity Strength, J. Graph Theory 41(2) (2002) 120-137.

[14] S. Janson, T. Łuczak, A. Ruciński, Random Graphs, Wiley, New York, 2000. 
[15] M. Kalkowski, A note on 1,2-Conjecture, in Ph.D. Thesis, 2009. Available at: https://repozytorium.amu.edu.pl/bitstream/10593/445/1 /Rozprawa\%20Doktorska\%202010\%20Maciej\%20Kalkowski.pdf

[16] M. Kalkowski, M. Karoński, F. Pfender, A new upper bound for the irregularity strength of graphs, SIAM J. Discrete Math. 25 (2011) 1319-1321.

[17] F. Kramer, H. Kramer, A survey on the distance-colouring of graphs, Discrete Math. 308 (2008) $422-426$.

[18] J. Lehel, Facts and quests on degree irregular assignments, Graph Theory, Combinatorics and Applications, Willey, New York, 1991, 765-782.

[19] P. Majerski, J. Przybyło, On the irregularity strength of dense graphs, SIAM J. Discrete Math. 28(1) (2014) 197-205.

[20] P. Majerski, J. Przybyło, Total vertex irregularity strength of dense graphs, J. Graph Theory 76(1) (2014) 34-41.

[21] T. Nierhoff, A tight bound on the irregularity strength of graphs, SIAM J. Discrete Math. 13(3) (2000) 313-323.

[22] Nurdin, E.T. Baskoro, A.N.M. Salman, N.N. Gaos, On the total vertex irregularity strength of trees, Discrete Math. 310(21) (2010) 3043-3048.

[23] J. Przybyło, Distant irregularity strength of graphs, Discrete Math. 313 (2013) 2875-2880.

[24] J. Przybyło, Distant irregularity strength of graphs with bounded minimum degree, submitted.

[25] J. Przybyło, Irregularity strength of regular graphs, Electron. J. Combin. 15(1) (2008) $\sharp R 82$.

[26] J. Przybyło, Linear bound on the irregularity strength and the total vertex irregularity strength of graphs, SIAM J. Discrete Math. 23(1) (2009) 511-516.

[27] J. Przybyło, M. Woźniak, On a 1,2 Conjecture, Discrete Math. Theor. Comput. Sci. 12:1 (2010) 101-108.

[28] J. Przybyło, M. Woźniak, Total weight choosability of graphs, Electron. J. Combin. 18(1) (2011) $\sharp \mathrm{P} 112$.

[29] R. Ramdani, A.N.M. Salman, H. Assiyatun, A. Semaničová-Feňovčiková, M. Bača, Total Irregularity Strength of Three Families of Graphs, Mathematics in Computer Science 9(2) (2015) 229-237.

[30] T. Wong, X. Zhu, Every graph is (2,3)-choosable, Combinatorica 36 (2016) 121-127.

[31] T. Wong, X. Zhu, Total weight choosability of graphs, J. Graph Theory 66 (2011) 198-212. 